Check for updates

Cite this: RSC Adv., 2020, 10, 739

Received 21st November 2019 Accepted 16th December 2019

DOI: $10.1039 / \mathrm{c} 9 \mathrm{ra0} 0745 \mathrm{~g}$

rsc.li/rsc-advances

\section{Diels-Alder reactions between cyclopentadiene analogs and benzoquinone in water and their application in the synthesis of polycyclic cage compounds $\dagger$}

\author{
Yijun Shi, (D) *ab Xuejing Liu, ${ }^{\text {*a }}$ Ying Han, ${ }^{a}$ Peng Yan, ${ }^{a}$ Fusheng Bie ${ }^{a}$ and Han $\mathrm{CaO}^{a}$ \\ Diels-Alder reactions between cyclopentadiene analogs and $p$-benzoquinone were explored in water and \\ yielded $83-97 \%$ product, higher than the results reported in water with a catalyst or cetrimonium bromide \\ (CTAB) micelles. The novel adduct 10 was synthesized and further used to synthesize the bi-cage \\ hydrocarbon 4,4'-spirobi[pentacyclo[5.4.0.0 $0^{2,6} \cdot 0^{3,10} \cdot 0^{5,9}$ ]undecane], which has a high density \\ $\left(1.2663 \mathrm{~g} \mathrm{~cm}^{-3}\right)$ and a high volumetric heat of combustion $\left(53.353 \mathrm{MJ} \mathrm{L}^{-1}\right)$. Four novel bi-cage \\ hydrocarbon compounds were synthesized in water using this method starting from 2,2'-bi( $p$ - \\ benzoquinone) and cyclopentadiene analogs.
}

\section{Introduction}

In recent years, water has been reported as a desirable solvent for many chemical reactions for reasons of cost, safety, and environmental concerns. Most notably, the Diels-Alder reaction has been found to be accelerated in dilute aqueous solution, and it is undoubtedly one of the most important reactions in the synthesis of polycyclic cage compounds. ${ }^{1-5}$ Polycyclic cage compounds have drawn more and more attention in the fields of medicinal chemistry and high energy density materials. ${ }^{6-56}$ The Diels-Alder reaction was developed by Diels and Alder in 1928 and the product of this reaction between cyclopentadiene and $p$-benzoquinone ${ }^{57}$ was further applied in the preparation of pentacyclo $\left[5 \cdot 4 \cdot 0 \cdot 0^{2,6} \cdot 0^{3,10} \cdot 0^{5,9}\right]$ undecane (PCUD) by Marchand ${ }^{53}$ (Scheme 1).

So far, several Diels-Alder reactions between cyclopentadiene analogs and $p$-benzoquinone have been carried out in water (Table 1) and the Diels-Alder adducts (Scheme 2) have been further applied in the synthesis of polycyclic cage compounds, but this required the addition of an organotungsten Lewis acid as a catalyst (entry 1$)^{58}$ or cetrimonium bromide (CTAB) micelles (entry 2$)^{59}$ and yielded lower than in organic solvents. Only the Diels-Alder reaction between 1,3cyclohexadiene and $p$-benzoquinone was done in water without

${ }^{a}$ Engineering and Technology Research Institute of Lunan Coal Chemical, Zaozhuang University, Beian Road, Zaozhuang 277160, Shandong Province, China. E-mail: 673230386@qq.com

${ }^{b}$ College of Chemistry, Chemical Engineering and Materials Science, Zaozhuang University, Beian Road, Zaozhuang 277160, Shandong Province, China

$\dagger$ Electronic supplementary information (ESI) available. CCDC 1936540, 1936541 and 1936543. For ESI and crystallographic data in CIF or other electronic format see DOI: $10.1039 / \mathrm{c} 9 \mathrm{ra} 09745 \mathrm{~g}$ a catalyst or CTAB micelles, but the yield was $67 \%$, which was lower than in toluene (entry 3). ${ }^{60}$ Apart from these, the other Diels-Alder reactions shown in Table 1 were done in organic solvents, and their yields were $76-97 \% .^{54,56,61-63}$

In this paper, Diels-Alder reactions between cyclopentadiene analogs and $p$-benzoquinone were further explored in water to improve the adduct yields. The new structure of adduct $\mathbf{1 0}$ is reported and used to synthesize the bi-cage hydrocarbon 4, $4^{\prime}$ spirobi[pentacyclo[5.4.0.0 $\left.2,6 \cdot 0^{3,10} \cdot 0^{5,9}\right]$ undecane], which has a high density $\left(1.2663 \mathrm{~g} \mathrm{~cm}^{-3}\right)$ and a high volumetric heat of combustion (53.353 $\left.\mathrm{MJ} \mathrm{L}^{-1}\right) .{ }^{56}$ Furthermore, new polycyclic bicage scaffolds were synthesized in water using this method starting from 2,2'-bi( $p$-benzoquinone) and cyclopentadiene analogs (1 and 2).

\section{Results and discussion}

In order to improve the yields of Diels-Alder reactions between cyclopentadiene analogs and $p$-benzoquinone in water, the reaction between cyclopentadiene and $p$-benzoquinone at room temperature in water was chosen as the model reaction. We found that dissolving $p$-benzoquinone into cyclopentadiene was an instantly exothermic process, and the state of this reaction changed to solid from liquid along with a decrease in temperature.

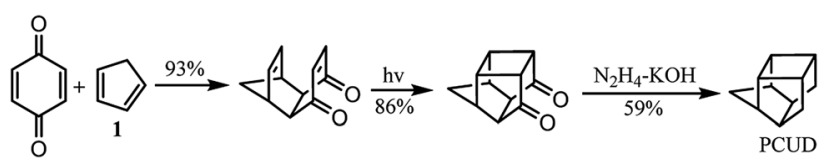

Scheme 1 Synthesis of PCUD. 
Table 1 The reported Diels-Alder reactions between cyclopentadiene analogs and $p$-benzoquinone

\begin{tabular}{|c|c|c|c|c|c|}
\hline Entry & Diene & Solvent & Conditions & Time/h & Yield/\% \\
\hline \multirow[t]{2}{*}{1} & 1 & $\mathrm{CH}_{2} \mathrm{Cl}_{2}$ & $0-25^{\circ} \mathrm{C}$, organotungsten Lewis acid, $\mathrm{rt}$ & 2.25 & $94-97$ \\
\hline & & Water & & 2 & 87 \\
\hline & & Water & & 3 & 66 \\
\hline \multirow[t]{2}{*}{3} & 3 & Toluene & Reflux, rt & 24 & 76 \\
\hline & & Water & & 48 & 67 \\
\hline
\end{tabular}

Based on this phenomenon and the solubility of $p$-benzoquinone in water, different methods of dropping were explored (Fig. 1). When water was added to $p$-benzoquinone (a), only a small amount of $p$-benzoquinone was dissolved in water (b). The system presented three phases because of the different densities after cyclopentadiene was added (c), and a large amount of $p$-benzoquinone was unreacted with cyclopentadiene after $24 \mathrm{~h}(\mathrm{~d})$.

When cyclopentadiene was added to $p$-benzoquinone (A), the state of this reaction changed to liquid from solid along with an increase in temperature. The system presented two liquid phases after water was added (C), and almost all of the product (6) had precipitated after $2 \mathrm{~h}$. The product was simply obtained in $96 \%$ yield by filtration and recrystallization. Compared with the reaction in Table 1 , this reaction was done in water without a catalyst and the yield was improved from $87 \%$ to $96 \%$. Compared with the method of dropping in Fig. $1 \mathrm{a}$ and $\mathrm{b}$, the method of dropping in Fig. 1A and B yielded more because it reacted more fully in two phases.

Based on the method shown in Fig. 1A-D, the results of DielsAlder reactions between cyclopentadiene analogs and $p$-benzoquinone in water are shown in Table 2 . The yields (90-97\%, entries 1-4) were higher than those reported previously and the novel product 10 was synthesized in $83 \%$ yield. Products 6-9 have been reported to be important in the synthesis of polycyclic cage hydrocarbons, such as PCUD and bi-cage hydrocarbon $4,4^{\prime}$-spirobi[pentacyclo[5.4.0.0 $\left.0^{2,6} \cdot 0^{3,10} \cdot 0^{5,9}\right]$ undecane].

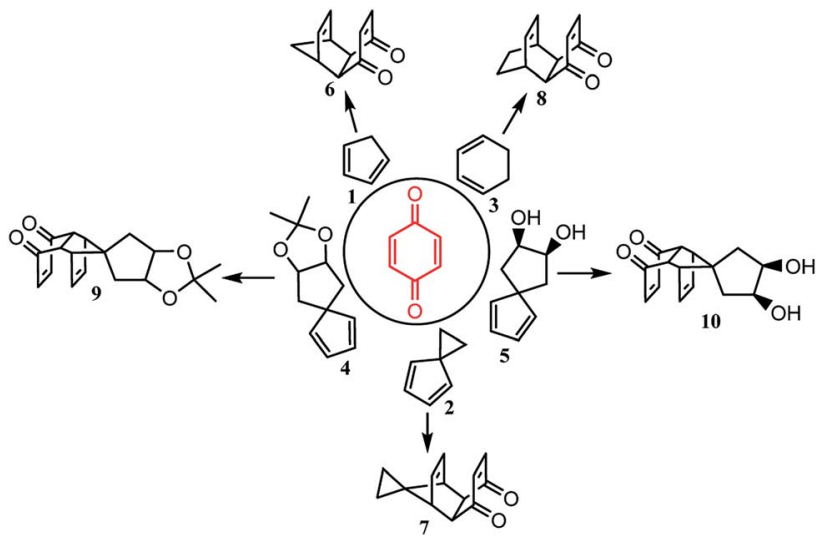

Scheme 2 Diels-Alder reactions between cyclopentadiene analogs and $p$-benzoquinone.
Further investigation was carried out to study the synthesis of a bi-cage hydrocarbon from adduct 10. Finally, the polycyclic cage hydrocarbon compound $\mathbf{1 2}$, which was important in the synthesis of $4,4^{\prime}$-spirobi[pentacyclo $\left[5 \cdot 4 \cdot 0.0^{2,6} \cdot 0^{3,10} \cdot 0^{5,9}\right]$ undecane], was synthesized by intramolecular $[2+2]$ photocyclization and Wolff-Kishner reduction from 10 (Scheme 3).

To expand the scope of this method in water, we found that the structure of 2,2'-bi( $p$-benzoquinone) was similar to that of $p$ benzoquinone and can be synthesized by oxidation of $2,2^{\prime}$ biphenol. ${ }^{64}$ According to the structure of $2,2^{\prime}$-bi( $p$-benzoquinone) and the high reactivity of $p$-benzoquinone with cyclopentadiene, inspiration was drawn and a convenient synthetic strategy to construct two cages simultaneously in the synthesis of bi-cage hydrocarbon compounds was designed (Scheme 4). In this scheme, the Diels-Alder reaction in water was applied and bi-cage hydrocarbon compounds HV-1 and HV-2 were synthesized.

Following Scheme 4, two isomers (D-A-1 : D-A-2 = $32: 28)$ were obtained by the Diels-Alder reaction of 2,2'-bi( $p$-benzoquinone) with cyclopentadiene with an overall yield of $60 \%$ and separated. The intramolecular $[2+2]$ photocyclization of D-A-1 at room temperature with a medium-pressure mercury immersion lamp formed $\mathbf{H V}-\mathbf{1}$ in $90 \%$ yield and $\mathbf{H V}-2$ was obtained in $87 \%$ yield by the intramolecular $[2+2]$ photocyclization of D-A-2.

To confirm the structures of the bi-cage hydrocarbon compounds HV-1 and HV-2, crystals of compounds HV-1 and

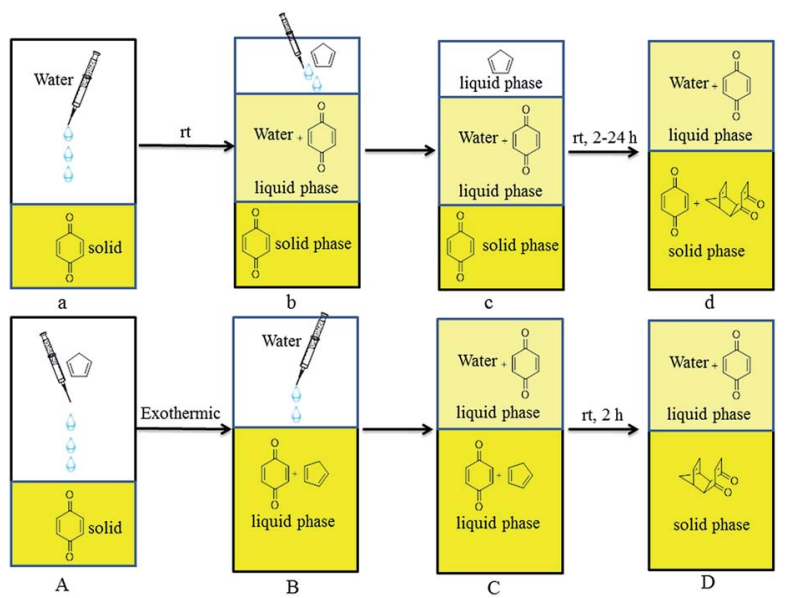

Fig. 1 Diels-Alder reactions between cyclopentadiene and $p$-benzoquinone $(1: 1)$ in water. 
Table 2 Diels-Alder reactions between cyclopentadiene analogs and $p$-benzoquinone in water ${ }^{a}$

\begin{tabular}{llllll}
\hline Entry & Diene & Solvent & Time $/ \mathrm{h}$ & Product & Yield $^{b} \%$ \\
\hline 1 & $\mathbf{1}$ & Water & 2 & $\mathbf{6}$ & 96 \\
2 & $\mathbf{2}$ & Water & 4 & 7 & 90 \\
3 & $\mathbf{3}$ & Water & 4 & $\mathbf{8}$ & 97 \\
4 & $\mathbf{4}$ & Water & 4 & $\mathbf{9}$ & 91 \\
5 & $\mathbf{5}$ & Water & 4 & $\mathbf{1 0}$ & 83
\end{tabular}

${ }^{a}$ Reaction conditions: $n$ (diene) $: n(p$-benzoquinone $)=1: 1, n(p$ benzoquinone $)=4.63 \mathrm{mmol}, V($ water, $\mathrm{pH}=7)=5 \mathrm{~mL}, \mathrm{rt}^{b}{ }^{b}$ Isolated yield.

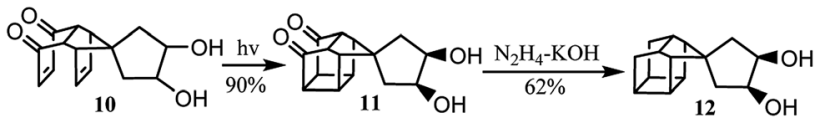

Scheme 3 Synthesis of 12 from 10.

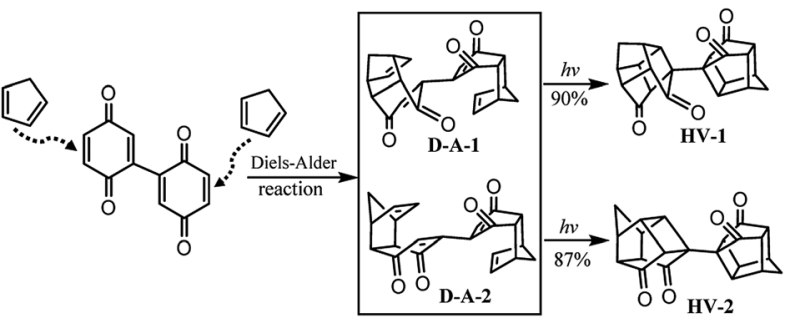

Scheme 4 Synthesis of bi-cage hydrocarbon compounds HV-1 and $\mathrm{HV}-2$.

HV-2 suitable for X-ray diffraction were obtained by recrystallization. In the crystal structures of compounds HV-1 and HV-2 (Fig. 2), two polycyclic cages were clearly connected with a single $\mathrm{C}-\mathrm{C}$ bond, which directly confirmed the bi-cage structures.

Based on Scheme 4, bi-cage hydrocarbon compounds HV-3 and HV-4 were synthesized as shown in Scheme 5. Two isomers (D-A-3 : D-A-4 = $38: 34$ ) were obtained by the Diels-Alder reaction of $2,2^{\prime}$-bi( $p$-benzoquinone) with 2 with an overall yield of $72 \%$ and separated. The intramolecular $[2+2]$ photocyclization of D-A-3 at room temperature with a medium-pressure mercury immersion lamp formed $\mathbf{H V}-\mathbf{3}$ in $88 \%$ yield and $\mathbf{H V}-\mathbf{4}$ was obtained in $80 \%$ yield by the intramolecular $[2+2]$ photocyclization of D-A-4.
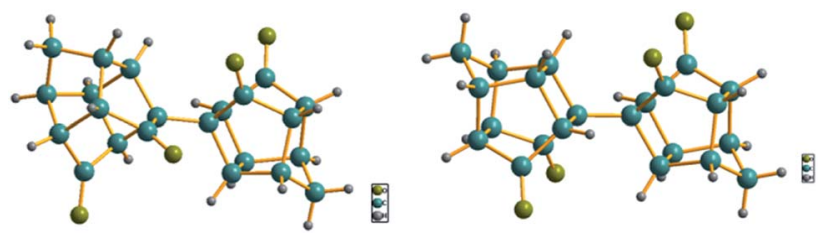

Fig. 2 X-ray crystal structures of HV-1 (CCDC 1936540) and HV-2 (CCDC 1936541).

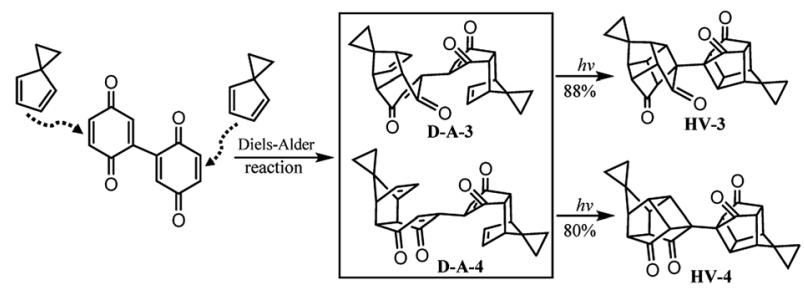

Scheme 5 Synthesis of bi-cage hydrocarbon compounds HV-3 and HV-4.

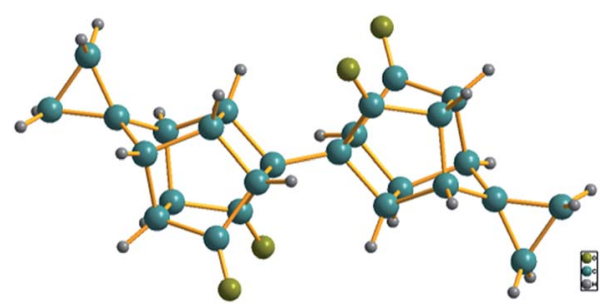

Fig. 3 X-ray crystal structure of HV-4 (CCDC 1936543).

To confirm the structures of the bi-cage hydrocarbon compounds HV-3 and HV-4, only a crystal of compound HV-4 suitable for X-ray diffraction was obtained by recrystallization. The crystal structure of compound HV-4 (Fig. 3) is very similar to the structure of compound $\mathbf{H V}-\mathbf{2}$ and the two cyclopropyl structures share two spiro-carbons with the bi-cage structures.

\section{Conclusions}

In summary, the Diels-Alder reactions between cyclopentadiene analogs and $p$-benzoquinone were done in water used the method of dropping shown in Fig. 1A and B and yielded more than previously reported in water with a catalyst or CTAB micelles. The novel adduct $\mathbf{1 0}$ was synthesized and further used to synthesize the bi-cage hydrocarbon 4,4'- spirobi[pentacyclo $\left[5.4 .0 .0^{2,6} \cdot 0^{3,10} \cdot 0^{5,9}\right]$ undecane]. Furthermore, four bi-cage hydrocarbon compounds were synthesized in water using this method starting from $2,2^{\prime}$-bi( $p$-benzoquinone) and cyclopentadiene analogs (1 and 2). Additionally, further work on the synthesis of more polycyclic cage (one cage or two cages) compounds is underway in our laboratory.

\section{Experimental}

\section{General procedures}

All chemicals were purchased from commercial sources and used without further purification. Melting points were measured using an INESA (SGWX-4B) melting point detector and are uncorrected. NMR spectra were recorded with Bruker Avance $400 \mathrm{M}$ or Bruker Avance 500M spectrometers. MS data were recorded with a Micromass-GC/TOF mass spectrometer GCT (UK) and a Thermo-LTQ ORBITRAP XL. IR data were recorded with a NICOLET 6700 FT-IR. The X-ray single-crystal diffraction analysis was performed on an Agilent Supernova CCD diffractometer instrument. 


\section{Synthesis and analytical data for 2}

2 was prepared by the procedure described by Marchand. ${ }^{54}{ }^{1} \mathrm{H}$ NMR (400 MHz, $\left.\mathrm{CDCl}_{3}\right) \delta$ 6.59-6.49 (m, 2H), 6.19-6.10 (m, 2H), $1.66(\mathrm{~s}, 4 \mathrm{H})$.

\section{Synthesis and analytical data for 4}

4 was prepared by the procedure described by Semmelhack. ${ }^{65}$ ${ }^{1} \mathrm{H}$ NMR (500 MHz, $\left.\mathrm{CDCl}_{3}\right) \delta$ 6.86-6.78 (m, 1H), 6.30-6.27 (m, $1 \mathrm{H})$, 6.21-6.14 (m, 1H), 6.08-5.99 (m, 1H), 4.88-4.81 (m, 2H), 2.12-2.03 (m, 2H), 1.77-1.72 (m, 2H), 1.60 (s, 3H), 1.34 (s, 3H).

\section{Synthesis and analytical data for 5}

5 was prepared by the procedure described by Semmelhack. ${ }^{65}$ ${ }^{1} \mathrm{H}$ NMR (400 MHz, $\left.\mathrm{CDCl}_{3}\right) \delta$ 6.39-6.35 (m, 1H), 6.25-6.20 (m, $1 \mathrm{H}), 6.20-6.13(\mathrm{~m}, 2 \mathrm{H}), 4.29(\mathrm{t}, J=4.4 \mathrm{~Hz}, 2 \mathrm{H}), 3.67(\mathrm{~s}, 1 \mathrm{H}), 3.60$ (s, 1H), 1.99-1.94 (m, 4H).

\section{Synthesis and analytical data for $2,2^{\prime}$-bi( $p$-benzoquinone)}

$2,2^{\prime}$-bi( $p$-benzoquinone) was prepared by the procedure described by Bouaziz. ${ }^{64}{ }^{1} \mathrm{H}$ NMR (400 MHz, $\left.\mathrm{CDCl}_{3}\right) \delta 6.85(\mathrm{~d}, J=$ $15.8 \mathrm{~Hz}, 6 \mathrm{H})$.

\section{Synthesis and analytical data for 6}

$0.50 \mathrm{~g}$ ( $4.63 \mathrm{mmol})$ of $p$-benzoquinone and $0.31 \mathrm{~g}(4.70 \mathrm{mmol})$ of cyclopentadiene were added sequentially to a $25 \mathrm{~mL}$ flask equipped with a magnetic stirrer bar. Water $(5 \mathrm{~mL})$ was added and the mixture was stirred at room temperature for $2 \mathrm{~h}$. The precipitate was filtered and recrystallized from $n$-hexane to yield 6 (0.77 g, $4.42 \mathrm{mmol}, 96 \%)$ as yellow needles. Mp: $76-77{ }^{\circ} \mathrm{C}$. IR (film): 2986, 2950, 2926, 1659, 1604, 1298, 1280, 1141, 1066, 915, 872, 852, 725, $709 \mathrm{~cm}^{-1} .{ }^{1} \mathrm{H}$ NMR (400 MHz, $\left.\mathrm{CDCl}_{3}\right) \delta 6.55(\mathrm{~s}$, $2 \mathrm{H}), 6.05(\mathrm{~d}, J=1.3 \mathrm{~Hz}, 2 \mathrm{H}), 3.53(\mathrm{~d}, J=1.1 \mathrm{~Hz}, 2 \mathrm{H}), 3.20(\mathrm{~d}, J=$ $1.2 \mathrm{~Hz}, 2 \mathrm{H}), 1.52(\mathrm{dd}, J=8.7,1.4 \mathrm{~Hz}, 1 \mathrm{H}), 1.41$ (d, $J=8.7 \mathrm{~Hz}$, 1H). ${ }^{13} \mathrm{C}$ NMR (101 MHz, $\left.\mathrm{CDCl}_{3}\right) \delta 199.46(\mathrm{C}), 142.06(\mathrm{CH})$, $135.30(\mathrm{CH}), 48.77(\mathrm{CH}), 48.71(\mathrm{CH}), 48.34\left(\mathrm{CH}_{2}\right)$.

\section{Synthesis and analytical data for 7}

7 was prepared with the same procedure as 6 starting with $p$ benzoquinone $(0.50 \mathrm{~g}, 4.63 \mathrm{mmol}), 2(0.44 \mathrm{~g}, 4.78 \mathrm{mmol})$ and water $(5 \mathrm{~mL})$. The mixture was stirred at room temperature for $4 \mathrm{~h}$. The precipitate was filtered and recrystallized from $n$ hexane to yield $7(0.83 \mathrm{~g}, 4.15 \mathrm{mmol}, 90 \%)$ as yellow needles. Mp: $100-102{ }^{\circ} \mathrm{C}$. IR (film): 2980, 1662, 1606, 1301, 1282, 1131, 1028, 956, 929, 897, 874, 848, $734 \mathrm{~cm}^{-1} \cdot{ }^{1} \mathrm{H}$ NMR $(400 \mathrm{MHz}$, $\left.\mathrm{CDCl}_{3}\right) \delta 6.58(\mathrm{~s}, 2 \mathrm{H}), 6.14(\mathrm{~d}, J=1.6 \mathrm{~Hz}, 2 \mathrm{H}), 3.38(\mathrm{~d}, J=1.4 \mathrm{~Hz}$, $2 \mathrm{H}$ ), 2.89 (s, 2H), 0.60 (dd, $J=9.3,5.7 \mathrm{~Hz}, 2 \mathrm{H}), 0.49$ (dd, $J=9.5$, $5.9 \mathrm{~Hz}, 2 \mathrm{H}) .{ }^{13} \mathrm{C}$ NMR (101 MHz, $\left.\mathrm{CDCl}_{3}\right) \delta 199.31$ (C), 142.32 $(\mathrm{CH}), 135.34(\mathrm{CH}), 53.66(\mathrm{C}), 49.21(\mathrm{CH}), 44.56(\mathrm{CH}), 7.96\left(\mathrm{CH}_{2}\right)$, $6.94\left(\mathrm{CH}_{2}\right)$.

\section{Synthesis and analytical data for 8}

8 was prepared with the same procedure as 6 starting with $p$ benzoquinone (0.50 g, $4.63 \mathrm{mmol}), 3(0.38 \mathrm{~g}, 4.75 \mathrm{mmol})$ and water $(5 \mathrm{~mL})$. The mixture was stirred at room temperature for
$4 \mathrm{~h}$. The precipitate was filtered and recrystallized from $n$ hexane to yield $8(0.84 \mathrm{~g}, 4.47 \mathrm{mmol}, 97 \%)$ as yellow needles. Mp: 88-90 ${ }^{\circ}$ C. IR (film): 2947, 2866, 1662, 1611, 1289, 1277, 1257, 1176, 1163, 1107, 1044, 1018, 877, 798, 722, $710 \mathrm{~cm}^{-1} \cdot{ }^{1} \mathrm{H}$ NMR (400 MHz, $\left.\mathrm{CDCl}_{3}\right) \delta 6.63(\mathrm{~s}, 2 \mathrm{H}), 6.29-6.11(\mathrm{~m}, 2 \mathrm{H}), 3.20(\mathrm{~d}$, $J=1.1 \mathrm{~Hz}, 2 \mathrm{H}), 2.98(\mathrm{~s}, 2 \mathrm{H}), 1.80-1.60$ (m, 2H), 1.47-1.17 (m, 2H). ${ }^{13} \mathrm{C}$ NMR (101 MHz, $\left.\mathrm{CDCl}_{3}\right) \delta 198.36(\mathrm{C}), 140.98(\mathrm{CH})$, $132.45(\mathrm{CH}), 48.32(\mathrm{CH}), 34.32(\mathrm{CH}), 23.72\left(\mathrm{CH}_{2}\right)$.

\section{Synthesis and analytical data for 9}

9 was prepared with the same procedure as 6 starting with $p$ benzoquinone $(0.50 \mathrm{~g}, 4.63 \mathrm{mmol}), 4(0.89 \mathrm{~g}, 4.63 \mathrm{mmol})$ and water $(5 \mathrm{~mL})$. The mixture was stirred at room temperature for $4 \mathrm{~h}$. The precipitate was filtered and recrystallized from $n$ hexane to yield $9(1.26 \mathrm{~g}, 4.20 \mathrm{mmol}, 91 \%)$ as yellow needles. Mp: 158-160 ${ }^{\circ} \mathrm{C}$. IR (film): 2974, 2943, 2908, 1666, 1604, 1384, 1372, 1283, 1208, 1119, 1052, 1022, 988, 877, 843, $728 \mathrm{~cm}^{-1} \cdot{ }^{1} \mathrm{H}$ NMR (400 MHz, $\left.\mathrm{CDCl}_{3}\right) \delta 6.57(\mathrm{~s}, 2 \mathrm{H}), 6.04(\mathrm{ddd}, J=30.2$, 5.7, $2.8 \mathrm{~Hz}, 2 \mathrm{H}), 4.57$ (dt, $J=32.1,6.0 \mathrm{~Hz}, 2 \mathrm{H}), 3.67$ (s, 1H), $3.43(\mathrm{dd}, J$ $=8.4,4.2 \mathrm{~Hz}, 1 \mathrm{H}), 3.17(\mathrm{dd}, J=8.4,4.0 \mathrm{~Hz}, 1 \mathrm{H}), 2.96(\mathrm{~s}, 1 \mathrm{H}), 2.03$ (dd, $J=14.9,1.9 \mathrm{~Hz}, 1 \mathrm{H}), 1.75$ (dd, $J=14.6,1.6 \mathrm{~Hz}, 1 \mathrm{H}), 1.62-$ $1.47(\mathrm{~m}, 5 \mathrm{H}), 1.26$ (s, 3H). ${ }^{13} \mathrm{C}$ NMR $\left(101 \mathrm{MHz}, \mathrm{CDCl}_{3}\right) \delta 199.43$ (C), $199.23(\mathrm{C}), 142.58(\mathrm{CH}), 141.97(\mathrm{CH}), 136.89(\mathrm{CH}), 134.37$ $(\mathrm{CH}), 109.33(\mathrm{C}), 80.54(\mathrm{CH}), 79.65(\mathrm{CH}), 68.18(\mathrm{C}), 57.42\left(\mathrm{CH}_{2}\right)$, $53.66\left(\mathrm{CH}_{2}\right), 47.99(\mathrm{CH}), 47.69(\mathrm{CH}), 37.77(\mathrm{CH}), 36.68(\mathrm{CH})$, $26.36\left(\mathrm{CH}_{3}\right), 23.52\left(\mathrm{CH}_{3}\right)$.

\section{Synthesis and analytical data for 10}

10 was prepared with the same procedure as 6 starting with $p$ benzoquinone $(0.50 \mathrm{~g}, 4.63 \mathrm{mmol}), 5(0.71 \mathrm{~g}, 4.67 \mathrm{mmol})$ and water $(5 \mathrm{~mL})$. The mixture was stirred at room temperature for $4 \mathrm{~h}$. The precipitate was filtered and subjected to chromatography on silica gel to give $10(1.00 \mathrm{~g}, 3.84 \mathrm{mmol}, 83 \%)$ as yellow needles. Mp: $160-162^{\circ} \mathrm{C}$. IR (film): 3348, 2968, 1712, 1659, 1325, 1124, 1075, $956 \mathrm{~cm}^{-1} .{ }^{1} \mathrm{H} \mathrm{NMR}\left(400 \mathrm{MHz}, \mathrm{CDCl}_{3}\right) \delta 6.58(\mathrm{~s}, 2 \mathrm{H})$, $6.04(\mathrm{dd}, J=9.3,2.0 \mathrm{~Hz}, 2 \mathrm{H}), 4.04(\mathrm{~d}, J=35.0 \mathrm{~Hz}, 2 \mathrm{H}), 3.36$ (s, $1 \mathrm{H}), 3.27$ (ddd, $J=18.1,8.3,3.9 \mathrm{~Hz}, 2 \mathrm{H}), 3.11$ (s, 1H), 2.40 (s, $1 \mathrm{H}), 2.27$ (s, 1H), 1.85-1.60 (m, 4H). ${ }^{13} \mathrm{C} \mathrm{NMR}\left(101 \mathrm{MHz}, \mathrm{CDCl}_{3}\right)$ $\delta 199.40(\mathrm{C}), 199.26(\mathrm{C}), 142.45(\mathrm{CH}), 142.24(\mathrm{CH}), 136.18(\mathrm{CH})$, $135.59(\mathrm{CH}), 73.62(\mathrm{CH}), 72.86(\mathrm{CH}), 64.22(\mathrm{C}), 57.75\left(\mathrm{CH}_{2}\right)$, $56.80\left(\mathrm{CH}_{2}\right), 48.18(\mathrm{CH}), 47.99(\mathrm{CH}), 37.08(\mathrm{CH}), 36.76(\mathrm{CH})$. HRMS (EI): $\mathrm{C}_{15} \mathrm{H}_{16} \mathrm{O}_{4}[\mathrm{M}]^{+}$calcd, 260.1049; found, 260.1054.

\section{Synthesis and analytical data for 11}

A solution of 10 (1.00 g, $3.84 \mathrm{mmol})$ in EtOAc $(200 \mathrm{~mL})$ was irradiated with a $400 \mathrm{~W}$ medium-pressure mercury immersion lamp for $3 \mathrm{~h}$. The reaction mixture was concentrated in vacuo, and the residue was subjected to chromatography on silica gel to give 11 ( $0.90 \mathrm{~g}, 3.46 \mathrm{mmol}, 90 \%)$ as a white solid. Mp: 200$202{ }^{\circ} \mathrm{C}$. IR (film): 3343, 2952, 2925, 1732, 1194, 1076, $1049 \mathrm{~cm}^{-1}$. ${ }^{1} \mathrm{H}$ NMR (400 MHz, DMSO) $\delta 4.40(\mathrm{~s}, 2 \mathrm{H}), 3.85$ (s, 2H), 3.17 (s, $2 \mathrm{H}), 2.74(\mathrm{~s}, 4 \mathrm{H}), 2.53(\mathrm{~d}, J=38.4 \mathrm{~Hz}, 2 \mathrm{H}), 1.78-1.43(\mathrm{~m}, 4 \mathrm{H}) .{ }^{13} \mathrm{C}$ NMR (101 MHz, DMSO) $\delta 213.19(\mathrm{C}), 73.12(\mathrm{CH}), 73.02(\mathrm{CH})$, $59.80(\mathrm{C}), 54.50\left(\mathrm{CH}_{2}\right), 54.46\left(\mathrm{CH}_{2}\right), 54.43(\mathrm{CH}), 53.33(\mathrm{CH}), 43.90$ $(\mathrm{CH}), 43.87(\mathrm{CH}), 38.39(\mathrm{CH}), 38.34(\mathrm{CH}), 37.41(\mathrm{CH}), 33.64(\mathrm{CH})$. HRMS (EI): $\mathrm{C}_{15} \mathrm{H}_{16} \mathrm{O}_{4}[\mathrm{M}]^{+}$calcd, 260.1049; found, 260.1057. 


\section{Synthesis and analytical data for 12}

A solution of $11(1.00 \mathrm{~g}, 3.84 \mathrm{mmol})$ and hydrazine monohydrate (3.3 $\mathrm{mL}, 80 \%, 54.31 \mathrm{mmol})$ in diethylene glycol $(3 \mathrm{~mL})$ was heated at $135{ }^{\circ} \mathrm{C}$ for $20 \mathrm{~h}$ and the solution was distilled until the temperature of the distillate reached $190{ }^{\circ} \mathrm{C}$. Solid potassium hydroxide $(1.51 \mathrm{~g}, 26.96 \mathrm{mmol})$ was added portionwise and the solution was heated at $200{ }^{\circ} \mathrm{C}$ for $20 \mathrm{~h}$, allowed to cool, and diluted with water $(50 \mathrm{~mL})$. The precipitate was filtered and recrystallized from EtOAc to yield 12 ( $0.55 \mathrm{~g}, 2.37 \mathrm{mmol}, 62 \%)$ as a white solid. Mp: $156-158^{\circ} \mathrm{C}$. IR (film): 3266, 2933, 2857, 1088, $1031 \mathrm{~cm}^{-1}$. ${ }^{1} \mathrm{H}$ NMR (400 MHz, DMSO) $\delta 4.18$ (dd, $J=11.7$, $4.2 \mathrm{~Hz}, 2 \mathrm{H}), 3.85-3.68(\mathrm{~m}, 2 \mathrm{H}), 2.50(\mathrm{~d}, J=30.5 \mathrm{~Hz}, 4 \mathrm{H}), 2.28$ (s, $2 \mathrm{H}), 1.88-1.60(\mathrm{~m}, 4 \mathrm{H}), 1.47-1.26(\mathrm{~m}, 4 \mathrm{H}), 0.86(\mathrm{~d}, J=11.9 \mathrm{~Hz}$, 2H). ${ }^{13} \mathrm{C}$ NMR (101 MHz, DMSO) $\delta 73.34(\mathrm{CH}), 73.26(\mathrm{CH}), 57.17$ $\left(\mathrm{CH}_{2}\right), 56.11\left(\mathrm{CH}_{2}\right), 51.80(\mathrm{C}), 43.12(\mathrm{CH}), 42.97(\mathrm{CH}), 41.33(\mathrm{CH})$, $41.16(\mathrm{CH}), 38.58(\mathrm{CH}), 37.48(\mathrm{CH}), 35.39(\mathrm{CH}), 35.35(\mathrm{CH}), 27.14$ $\left(\mathrm{CH}_{2}\right), 27.06\left(\mathrm{CH}_{2}\right)$.

\section{Synthesis and analytical data for D-A-1 and D-A-2}

$0.50 \mathrm{~g}$ (2.34 mmol) 2,2'-bi( $p$-benzoquinone) and $0.31 \mathrm{~g}$ (4.70 $\mathrm{mmol}$ ) cyclopentadiene were added one after another to a $25 \mathrm{~mL}$ flask equipped with a magnetic stirrer bar. Water $(5 \mathrm{~mL})$ was added and the mixture was stirred at room temperature for $4 \mathrm{~h}$. The precipitate was filtered and subjected to chromatography on silica gel to give D-A-1 (0.26 g, $0.75 \mathrm{mmol}, 32 \%)$ as a yellow microcrystalline solid and D-A-2 $(0.23 \mathrm{~g}, 0.66 \mathrm{mmol}, 28 \%)$ as a yellow microcrystalline solid.

D-A-1. Mp: $162-164{ }^{\circ} \mathrm{C}$. IR (film): 2984, 2958, 1671, 1658, 1197, 1091, 1044, 903, 800, 734, $718 \mathrm{~cm}^{-1} \cdot{ }^{1} \mathrm{H}$ NMR $(400 \mathrm{MHz}$, $\left.\mathrm{CDCl}_{3}\right) \delta 6.43(\mathrm{~s}, 2 \mathrm{H}), 6.21(\mathrm{dd}, J=5.4,2.9 \mathrm{~Hz}, 2 \mathrm{H}), 6.05(\mathrm{dd}, J=$ 5.5, $2.9 \mathrm{~Hz}, 2 \mathrm{H}), 3.52(\mathrm{~s}, 4 \mathrm{H}), 3.37-3.23(\mathrm{~m}, 4 \mathrm{H}), 1.58-1.52(\mathrm{~m}$, $2 \mathrm{H}), 1.43(\mathrm{~d}, J=8.8 \mathrm{~Hz}, 2 \mathrm{H}) .{ }^{13} \mathrm{C} \mathrm{NMR}\left(101 \mathrm{MHz}, \mathrm{CDCl}_{3}\right) \delta 197.47$ (C), 195.86 (C), 147.25 (C), 138.91 (CH), 134.87 (CH), 134.07 $(\mathrm{CH}), 48.48(\mathrm{CH}), 48.09(\mathrm{CH}), 47.85(\mathrm{CH}), 47.75(\mathrm{CH})$. HRMS (ESI): $\mathrm{C}_{22} \mathrm{H}_{18} \mathrm{O}_{4}[\mathrm{M}+\mathrm{Na}]^{+}$calcd, 369.11028; found, 369.10995.

D-A-2. Mp: $158-160{ }^{\circ} \mathrm{C}$. IR (film): 2985, 2962, 2932, 1665, 1605, 1322, 1246, 1194, 1052, 843, 748, 730, $719 \mathrm{~cm}^{-1} \cdot{ }^{1} \mathrm{H}$ NMR $\left(400 \mathrm{MHz}, \mathrm{CDCl}_{3}\right) \delta 6.44(\mathrm{~s}, 2 \mathrm{H}), 6.25-6.17(\mathrm{~m}, 2 \mathrm{H}), 6.07$ (dd, $J=$ $5.2,2.3 \mathrm{~Hz}, 2 \mathrm{H}), 3.52(\mathrm{~s}, 4 \mathrm{H}), 3.31(\mathrm{ddd}, J=23.3,8.9,3.8 \mathrm{~Hz}, 4 \mathrm{H})$, 1.55 (d, $J=8.8 \mathrm{~Hz}, 2 \mathrm{H}), 1.43$ (d, $J=8.7 \mathrm{~Hz}, 2 \mathrm{H}) .{ }^{13} \mathrm{C} \mathrm{NMR}(101$ $\mathrm{MHz}, \mathrm{CDCl}_{3}$ ) $\delta 197.47(\mathrm{C}), 195.98(\mathrm{C}), 147.45(\mathrm{C}), 138.76(\mathrm{CH})$, $134.93(\mathrm{CH}), 134.14(\mathrm{CH}), 48.55(\mathrm{CH}), 48.13(\mathrm{CH}), 47.78(\mathrm{CH})$, $47.64(\mathrm{CH}), 47.54\left(\mathrm{CH}_{2}\right)$. HRMS (ESI): $\mathrm{C}_{22} \mathrm{H}_{18} \mathrm{O}_{4}[\mathrm{M}+\mathrm{Na}]^{+}$calcd, 369.11028; found, 369.10960.

\section{Synthesis and analytical data for $\mathrm{HV}-1$}

A solution of D-A-1 $(0.51 \mathrm{~g}, 1.47 \mathrm{mmol})$ in EtOAc $(30 \mathrm{~mL})$ was irradiated with a $400 \mathrm{~W}$ medium-pressure mercury immersion lamp for $2 \mathrm{~h}$. The reaction mixture was concentrated in vacuum, and the residue was subjected to chromatography on silica gel to give HV-1 (0.46 g, $1.33 \mathrm{mmol}, 90 \%)$ as a white solid. Mp: 310$313{ }^{\circ} \mathrm{C}$. IR (film): 2962, 2944, 1742, 1731, 1079, $1042 \mathrm{~cm}^{-1} \cdot{ }^{1} \mathrm{H}$ NMR (400 MHz, $\left.\mathrm{CDCl}_{3}\right) \delta 3.15(\mathrm{~d}, J=6.2 \mathrm{~Hz}, 2 \mathrm{H}), 2.99(\mathrm{~d}, J=$ $28.5 \mathrm{~Hz}, 6 \mathrm{H}), 2.71$ (d, $J=11.6 \mathrm{~Hz}, 6 \mathrm{H}), 2.03$ (d, $J=11.3 \mathrm{~Hz}, 2 \mathrm{H}$ ), $1.89(\mathrm{~d}, J=11.3 \mathrm{~Hz}, 2 \mathrm{H}) .{ }^{13} \mathrm{C}$ NMR $\left(101 \mathrm{MHz}, \mathrm{CDCl}_{3}\right) \delta 211.85$
(C), $210.54(\mathrm{C}), 55.17(\mathrm{C}), 55.08(\mathrm{CH}), 52.36(\mathrm{CH}), 46.26(\mathrm{CH})$, $44.72(\mathrm{CH}), 44.14(\mathrm{CH}), 42.50(\mathrm{CH}), 40.88(\mathrm{CH}), 37.47\left(\mathrm{CH}_{2}\right)$. HRMS (EI): $\mathrm{C}_{22} \mathrm{H}_{18} \mathrm{O}_{4}[\mathrm{M}]^{+}$calcd, 346.1205; found, 346.1212.

\section{Synthesis and analytical data for $\mathrm{HV}-2$}

A solution of D-A-2 (0.46 g, $1.33 \mathrm{mmol})$ in EtOAc $(30 \mathrm{~mL})$ was irradiated with a $400 \mathrm{~W}$ medium-pressure mercury immersion lamp for $2 \mathrm{~h}$. The reaction mixture was concentrated in vacuum, and the residue was subjected to chromatography on silica gel to give HV-2 (0.40 g, $1.16 \mathrm{mmol}, 87 \%)$ as white solid. Mp: 306$309{ }^{\circ}$ C. IR (film): 2991, 2935 1742, 1718, 1223, $1083 \mathrm{~cm}^{-1} \cdot{ }^{1} \mathrm{H}$ NMR (400 MHz, $\left.\mathrm{CD}_{2} \mathrm{Cl}_{2}\right) \delta 3.22-3.04(\mathrm{~m}, 2 \mathrm{H}), 3.03-2.73(\mathrm{~m}, 6 \mathrm{H})$, $2.62(\mathrm{~s}, 4 \mathrm{H}), 2.40(\mathrm{~s}, 2 \mathrm{H}), 1.96(\mathrm{~d}, J=11.2 \mathrm{~Hz}, 2 \mathrm{H}), 1.82(\mathrm{~d}, J=$ 11.2 Hz, 2H). ${ }^{13} \mathrm{C}$ NMR (101 MHz, $\left.\mathrm{CDCl}_{3}\right) \delta 210.66$ (C), 209.57 (C), $54.00(\mathrm{C}), 53.73(\mathrm{CH}), 50.81(\mathrm{CH}), 45.69(\mathrm{CH}), 43.23(\mathrm{CH})$, $42.85(\mathrm{CH}), 40.56(\mathrm{CH}), 39.85(\mathrm{CH}), 36.54\left(\mathrm{CH}_{2}\right)$. HRMS (EI): $\mathrm{C}_{22} \mathrm{H}_{18} \mathrm{O}_{4}[\mathrm{M}]^{+}$calcd, 346.1205; found, 346.1206.

\section{Synthesis and analytical data for D-A-3 and D-A-4}

D-A-3 and D-A-4 were prepared with the same procedure as D-A1 and D-A-2 starting with 2,2'-bi(p-benzoquinone) ( $0.50 \mathrm{~g}, 2.34$ $\mathrm{mmol}), 2(0.44 \mathrm{~g}, 4.78 \mathrm{mmol})$ and water $(5 \mathrm{~mL})$. The mixture was stirred at room temperature for $4 \mathrm{~h}$. The precipitate was filtered and subjected to chromatography on silica gel to give D-A-3 $(0.35 \mathrm{~g}, 0.88 \mathrm{mmol}, 38 \%)$ as a yellow microcrystalline solid and D-A-4 (0.32 g, $0.80 \mathrm{mmol}, 34 \%)$ as a yellow microcrystalline solid.

D-A-3. Mp: $163-165{ }^{\circ} \mathrm{C}$. IR (film): 2997, 1663, 1602, 1245 , 1227, 1029, 910, $730 \mathrm{~cm}^{-1} .{ }^{1} \mathrm{H}$ NMR $\left(400 \mathrm{MHz}, \mathrm{CDCl}_{3}\right) \delta 6.48(\mathrm{~s}$, $2 \mathrm{H}), 6.33-6.23$ (m, 2H), 6.19-6.08 (m, 2H), 3.48 (ddd, $J=19.9$, 8.5, 3.8 Hz, 4H), $2.90(\mathrm{~s}, 4 \mathrm{H}), 0.66-0.56(\mathrm{~m}, 4 \mathrm{H}), 0.55-0.44(\mathrm{~m}$, $4 \mathrm{H}) .{ }^{13} \mathrm{C} \mathrm{NMR}\left(101 \mathrm{MHz}, \mathrm{CDCl}_{3}\right) \delta 197.30(\mathrm{C}), 195.70(\mathrm{C}), 147.51$ (C), $139.14(\mathrm{CH}), 134.92(\mathrm{CH}), 134.18(\mathrm{CH}), 52.66(\mathrm{CH}), 52.57$ $(\mathrm{CH}), 49.37(\mathrm{CH}), 48.94(\mathrm{CH}), 43.71(\mathrm{C}), 6.97\left(\mathrm{CH}_{2}\right), 5.94\left(\mathrm{CH}_{2}\right)$. HRMS (ESI): $\mathrm{C}_{26} \mathrm{H}_{22} \mathrm{O}_{4}[\mathrm{M}+\mathrm{Na}]^{+}$calcd, 421.14103; found, 421.14098.

D-A-4. Mp: $156-158{ }^{\circ} \mathrm{C}$. IR (film): 2977, 1668, 1223, 1198, 1016, 930, 904, 766, 742, $729 \mathrm{~cm}^{-1} .{ }^{1} \mathrm{H}$ NMR (400 MHz, $\mathrm{CDCl}_{3}$ ) $\delta 6.48(\mathrm{~s}, 2 \mathrm{H}), 6.33-6.27(\mathrm{~m}, 2 \mathrm{H}), 6.20-6.15(\mathrm{~m}, 2 \mathrm{H}), 3.56-3.42$ $(\mathrm{m}, 4 \mathrm{H}), 2.97-2.79(\mathrm{~m}, 4 \mathrm{H}), 0.69-0.56(\mathrm{~m}, 4 \mathrm{H}), 0.54-0.43(\mathrm{~m}$, $4 \mathrm{H}) .{ }^{13} \mathrm{C} \mathrm{NMR}\left(101 \mathrm{MHz}, \mathrm{CDCl}_{3}\right) \delta 198.36(\mathrm{C}), 196.86(\mathrm{C}), 148.78$ (C), $140.03(\mathrm{CH}), 136.02(\mathrm{CH}), 135.24(\mathrm{CH}), 53.57(\mathrm{CH}), 53.47$ $(\mathrm{CH}), 50.46(\mathrm{CH}), 50.01(\mathrm{CH}), 44.70(\mathrm{C}), 7.98\left(\mathrm{CH}_{2}\right), 6.99\left(\mathrm{CH}_{2}\right)$. HRMS (ESI): $\mathrm{C}_{26} \mathrm{H}_{22} \mathrm{O}_{4}[\mathrm{M}+\mathrm{Na}]^{+}$calcd, 421.14103; found, 421.14130.

\section{Synthesis and analytical data for $\mathrm{HV}-3$}

HV-3 was prepared with same procedure as HV-1 and yielded in $88 \%$ as white solid. Mp: $260-262{ }^{\circ} \mathrm{C}$. IR (film): $2985,1734,1182$, 1080, 1048, 956, 942, 922, $876 \mathrm{~cm}^{-1} .{ }^{1} \mathrm{H}$ NMR (400 MHz, $\mathrm{CDCl}_{3}$ ) $\delta 3.31(\mathrm{dd}, J=13.9,6.2 \mathrm{~Hz}, 2 \mathrm{H}), 3.19-3.05(\mathrm{~m}, 2 \mathrm{H}), 2.93(\mathrm{~s}, 4 \mathrm{H})$, $2.79(\mathrm{~d}, J=6.2 \mathrm{~Hz}, 2 \mathrm{H}), 2.38(\mathrm{~s}, 2 \mathrm{H}), 2.32-2.23(\mathrm{~m}, 2 \mathrm{H}), 0.77-0.56$ $(\mathrm{m}, 8 \mathrm{H}) .{ }^{13} \mathrm{C}$ NMR (101 MHz, $\left.\mathrm{CDCl}_{3}\right) \delta 211.87$ (C), 210.52 (C), $55.63(\mathrm{CH}), 55.56(\mathrm{CH}), 53.11(\mathrm{CH}), 50.34(\mathrm{CH}), 49.68(\mathrm{CH}), 46.95$ (C), $42.72(\mathrm{C}), 37.70(\mathrm{CH}), 37.50(\mathrm{CH}), 5.50\left(\mathrm{CH}_{2}\right), 4.05\left(\mathrm{CH}_{2}\right)$. HRMS (EI): $\mathrm{C}_{26} \mathrm{H}_{22} \mathrm{O}_{4}[\mathrm{M}]^{+}$calcd, 398.1518; found, 398.1527. 


\section{Synthesis and analytical data for $\mathrm{HV}-4$}

HV-4 was prepared with the same procedure as $\mathbf{H V - 1}$ and yielded $80 \%$ as a white solid. Mp: $318-320{ }^{\circ} \mathrm{C}$. IR (film): 3003,2985 , 2962, 1727, 1221, 1181, 1083, 974, 955, 942, $761 \mathrm{~cm}^{-1} .{ }^{1} \mathrm{H}$ NMR $\left(400 \mathrm{MHz}, \mathrm{CDCl}_{3}\right) \delta 3.48-3.34(\mathrm{~m}, 2 \mathrm{H}), 3.24(\mathrm{~s}, 2 \mathrm{H}), 3.00-2.85$ (m, 4H), $2.43(\mathrm{~s}, 2 \mathrm{H}), 2.29-2.15(\mathrm{~m}, 4 \mathrm{H}), 0.77-0.56(\mathrm{~m}, 8 \mathrm{H}) .{ }^{13} \mathrm{C}$ NMR (101 MHz, $\left.\mathrm{CDCl}_{3}\right) \delta 210.65(\mathrm{C}), 209.48(\mathrm{C}), 54.48(\mathrm{CH})$, $54.18(\mathrm{CH}), 51.50(\mathrm{CH}), 48.86(\mathrm{CH}), 48.45(\mathrm{CH}), 46.36(\mathrm{C}), 40.77$ (C), $36.77(\mathrm{CH}), 36.52(\mathrm{CH}), 4.43\left(\mathrm{CH}_{2}\right), 3.06\left(\mathrm{CH}_{2}\right)$. HRMS (EI): $\mathrm{C}_{26} \mathrm{H}_{22} \mathrm{O}_{4}[\mathrm{M}]^{+}$calcd, 398.1518; found, 398.1522.

\section{Conflicts of interest}

There are no conflicts to declare.

\section{Notes and references}

1 N. Windmon and V. Dragojlovic, Green Chem. Lett. Rev., 2008, 1, 155-163.

2 P. A. Grieco, K. Yoshida and P. Garner, J. Org. Chem., 1983, 48, 3137-3139.

3 M. C. Pirrung and K. D. Sarma, Tetrahedron, 2005, 61, 1145611472.

4 Y. Jung and R. A. Marcus, J. Am. Chem. Soc., 2007, 129, 54925502.

5 S. Narayan, J. Muldoon, M. G. Finn, V. V. Fokin, H. C. Kolb and K. B. Sharpless, Angew. Chem., Int. Ed., 2005, 44, 32753279.

6 J. Wlochal, R. D. M. Davies and J. Burton, Org. Lett., 2014, 16, 4094.

7 W. J. Geldenhuys, S. F. Malan, T. Murugesan, C. J. Van der Schyf and J. R. Bloomquist, Bioorg. Med. Chem., 2004, 12, 1799.

8 W. J. Geldenhuys, S. F. Malan, J. R. Bloomquist, A. P. Marchand and C. Van der Schyf, Med. Res. Rev., 2005, 25, 21.

9 A. Mdzinarishvili, W. J. Geldenhuys, T. J. Abbruscato, U. Bickel, J. Klein and C. Van der Schyf, Neurosci. Lett., 2005, 383, 49.

10 D. W. Oliver and S. F. Malan, Med. Chem. Res., 2008, 17, 137.

11 H. Gunosewoyo, J. L. Guo, M. R. Bennett, M. J. Coster and M. Kassiou, Bioorg. Med. Chem. Lett., 2008, 18, 3720.

12 J. Hao, A. Mdzinarishvili, T. J. Abbruscato, J. Klein, W. J. Geldenhuys, C. J. Van der Schyf and U. Bickel, Brain Res., 2008, 1196, 113.

13 W. J. Geldenhuys, L. M. Bezuidenhout and D. E. Dluzen, Eur. J. Pharmacol., 2009, 619, 38.

14 C. J. Van der Schyf and W. J. Geldenhuys, Neurotherapeutics, 2009, 6, 175.

15 J. Joubert, S. van Dyk, I. R. Green and S. F. Malan, Eur. J. Med. Chem., 2011, 46, 5010.

16 J. Wang, C. Ma, V. Balannik, L. H. Pinto, R. A. Lamb and W. F. DeGrado, ACS Med. Chem. Lett., 2011, 2, 307.

17 H. J. R. Lemmer, J. Joubert, S. van Dyk, F. H. van der Westhuizen and S. F. Malan, Med. Chem., 2012, 8, 361.
18 J. Joubert, W. J. Geldenhuys, C. J. Van der Schyf, D. W. Oliver, H. G. Kruger, T. Govender and S. F. Malan, ChemMedChem, 2012, 7, 375.

19 J. A. Lockman, W. J. Geldenhuys, M. R. Jones-Higgins, J. D. Patrick, D. D. Allen and C. Van der Schyf, Brain Res., 2012, 1489, 133.

20 A. S. Sklyarova, V. N. Rodionov, C. G. Parsons, G. Quack, P. R. Schreiner and A. A. Fokin, Med. Chem. Res., 2013, 22, 360.

21 J. Joubert, R. Sharma, M. Onani and S. F. Malan, Tetrahedron Lett., 2013, 54, 6923.

22 C. Beinat, S. D. Banister, J. Hoban, J. Tsanaktsidis, A. Metaxas, A. D. Windhorst and M. Kassiou, Bioorg. Med. Chem. Lett., 2014, 24, 828.

23 J. Joubert, H. Samsodien, Q. R. Baber, D. L. Cruickshank, M. R. Caira and S. F. Malan, J. Chem. Crystallogr., 2014, 44, 194.

24 S. M. Wilkinson, H. Gunosewoyo, M. L. Barron, A. Boucher, M. McDonnell, P. Turner, D. E. Morrison, M. R. Bennett, I. S. McGregor, L. M. Rendina and M. Kassiou, ACS Chem. Neurosci., 2014, 5, 335.

25 F. T. Zindo, Q. R. Barber, J. Joubert, J. J. Bergh, J. P. Petzer and S. F. Malan, Eur. J. Med. Chem., 2014, 80, 122.

26 A. O. Egunlusi, S. F. Malan and J. Joubert, ChemMedChem, 2015, 10, 1259.

27 F. T. Zindo, J. Joubert and S. F. Malan, Future Med. Chem., 2015, 7, 609.

28 J. Joubert, E. Kapp, D. Taylor, P. J. Smith and S. F. Malan, Bioorg. Med. Chem. Lett., 2016, 26, 1151.

29 A. d. Meijere, S. Redlich, D. Frank, J. Magull, A. Hofmeister, H. Menzel, B. Konig and J. Svoboda, Angew. Chem., Int. Ed., 2007, 46, 4574.

30 K. F. Biegasiewicz, J. R. Griffiths, G. P. Savage, J. Tsanaktsidis and R. Priefer, Chem. Rev., 2015, 115, 6719.

31 H. N. Lim and G. Dong, Org. Lett., 2016, 18, 1104.

32 A. P. Marchand, M. N. Deshpande and G. M. Reddy, Trans. ASME: J. Energy Resour. Technol., 1989, 34, 946.

33 C. Segal and W. Shyy, Trans. ASME, 1996, 118, 180.

34 C. Segal, S. Pethe and K. R. Williams, Combust. Sci. Technol., 2001, 163, 229.

35 L. Qiu, D. Ye, W. Wei, K. Chen, J. Hou, J. Zheng, X. Gong and M. Xiao, J. Mol. Struct.: THEOCHEM, 2008, 866, 63.

36 S. Lal, S. Rajkumar, A. Tare, S. Reshmi, A. Chowdhury and I. N. N. Namboothiri, Chem.-Asian J., 2014, 9, 3533.

37 L. Pan, R. Feng, H. Peng, X. E, J. Zhou, L. Wang and X. Zhang, RSC Adv., 2014, 4, 50998.

38 C. Segal, M. J. Friedauer, H. S. Udaykumar, W. Shyy and A. P. Marchand, J. Propul. Power, 1997, 13, 246.

39 R. J. Stedman, L. S. Miller, L. D. Davis and J. R. E. Hoover, J. Org. Chem., 1970, 35, 4169.

40 A. P. Marchand and R. W. Allen, J. Org. Chem., 1974, 39, 1596.

41 P. G. Gassman and R. Yamaguchi, J. Org. Chem., 1978, 43, 4654.

42 A. P. Marchand, Chem. Rev., 1989, 89, 1011.

43 G. Mehta, S. Padma and S. R. Karra, J. Org. Chem., 1989, 54, 1342.

44 G. W. Griffin, Chem. Rev., 1989, 89, 997. 
45 M. A. Forman and W. P. Dailey, J. Org. Chem., 1993, 58, 1501. 46 H. Takeshita, H. Kawakami, Y. Ikeda and A. Mori, J. Org. Chem., 1994, 59, 6490.

47 A. d. Meijere, C. Lee, B. Bengtson, E. Pohl, S. I. Kozhushkov, P. R. Schreiner, R. Boese and T. Haumann, Chem.-Eur. J, 2003, 9, 5481.

48 A. M. Kenwright and J. D. Sellars, Magn. Reson. Chem., 2012, 50, 803.

49 A. P. Marchand, P. Jin and M. N. Deshpande, Acta Crystallogr., 1988, 44, 1617.

50 A. P. Marchand, G. M. Reddy, M. N. Deshpande, W. H. Watson, A. Nagl, O. S. Lee and E. Osawa, J. Am. Chem. Soc., 1990, 112, 3521.

51 A. P. Marchand, P. Jin and K. Siam, J. Mol. Struct.: THEOCHEM, 1990, 204, 209.

52 A. P. Marchand and V. Vidyasagar, J. Org. Chem., 1991, 56, 282.

53 A. P. Marchand, A. Zope, F. Zaragoza and G. Bott, Tetrahedron, 1994, 50, 1687.

54 A. P. Marchand, V. D. Sorokin, W. H. Watson, T. F. Carlson and M. Krawiec, Struct. Chem., 1994, 5, 367.
55 Y. Shi, J. Jiang, L. Ma, J. Wang and W. Li, Tetrahedron Lett., 2017, 58, 1376.

56 Y. Shi, J. Jiang, J. Wang and Y. Wang, Tetrahedron Lett., 2015, 56, 6704.

57 C. C. Nawrat and C. J. Moody, Angew. Chem., Int. Ed., 2014, 53, 2056.

58 I. Chen, J. Young and S. Yu, Tetrahedron, 2004, 60, 11903.

59 V. K. Singh, B. N. S. Raju and P. T. Deota, Synth. Commun., 1986, 16, 1731.

60 T. Sunakawa and C. Kuroda, Molecules, 2005, 10, 244.

61 O. Masaji, K. Takeshi, O. Tomoaki and E. Tetsuya, Org. Synth., 1996, 73, 253.

62 D. Mal and S. Ray, Eur. J. Org. Chem., 2008, 3014.

63 Y. Ishii, S. Ito, Y. Saito, D. Uno and T. Oba, Tetrahedron, 2015, 71, 8892.

64 Z. Bouaziz, A. Gherardi, F. Regnier, M. Sarciron, X. Bertheau, B. Fenet, N. Walchshofer and H. Fillion, Eur. J. Org. Chem., 2002, 1834.

65 M. F. Semmelhack, J. S. Foos and S. Katz, J. Am. Chem. Soc., 1973, 95, 7325. 